David MÁrquez-Carreras (Barcelona)

\title{
THE MAGNETIZATION AT HIGH TEMPERATURE FOR A $p$-SPIN INTERACTION MODEL WITH EXTERNAL FIELD
}

Abstract. This paper is devoted to a detailed and rigorous study of the magnetization at high temperature for a $p$-spin interaction model with external field, generalizing the Sherrington-Kirkpatrick model. In particular, we prove that $\left\langle\sigma_{i}\right\rangle$ (the mean of a spin with respect to the Gibbs measure) converges to an explicitly given random variable, and that $\left\langle\sigma_{1}\right\rangle, \ldots,\left\langle\sigma_{n}\right\rangle$ are asymptotically independent.

1. Introduction. We consider a spin glass model with the configuration space $\Sigma_{N}=\{-1,1\}^{N}$ where the energy of a given configuration $\sigma \in \Sigma_{N}$ is represented by a Hamiltonian $H(\sigma)$. We are interested in the Gibbs measure $G_{N}$ whose density with respect to the uniform measure $\mu_{N}$ on $\Sigma_{N}$ is $Z_{N}^{-1} e^{-H}$, where $Z_{N}$ is the normalization factor

$$
Z_{N}=\sum_{\sigma \in \Sigma_{N}} \exp (-H(\sigma)) .
$$

In order to introduce our model we borrow the notations of Bardina et al. (2004). The Hamiltonian of the $p$-spin interaction model with external field is defined by

$$
-H_{N, \beta, h}(\sigma)=\beta u_{N} \sum_{\left(i_{1}, \ldots, i_{p}\right) \in A_{N}^{p}} g_{i_{1}, \ldots, i_{p}} \sigma_{i_{1}} \ldots \sigma_{i_{p}}+h \sum_{i=1}^{N} \sigma_{i},
$$

with

$$
\begin{aligned}
& u_{N}=\left(\frac{p !}{2 N^{p-1}}\right)^{1 / 2}, \\
& A_{N}^{p}=\left\{\left(i_{1}, \ldots, i_{p}\right) \in \mathbb{N}^{p} ; 1 \leq i_{1}<\cdots<i_{p} \leq N\right\},
\end{aligned}
$$

2000 Mathematics Subject Classification: 82D30, 60G15, 60G60.

Key words and phrases: spin glasses, $p$-spin model, magnetization, cavity method.

Research partially supported by DGES grant BFM2003-01345. 
where the parameter $\beta$ represents the inverse of the temperature and where $g=\left\{g_{i_{1}, \ldots, i_{p}} ;\left(i_{1}, \ldots, i_{p}\right) \in A_{N}^{p}\right\}$ is a family of independent standard Gaussian random variables. The strictly positive parameter $h$ stands for the external magnetic field, under which the spins tend to take the same value +1 .

In physics, this kind of model was introduced to study the spin distribution of atoms submitted to disordered long range interactions (see, for instance, the paper of Gardner (1985)). In mathematics, the $p$-spin interaction model is a natural generalization of the SK model (see Sherrington and Kirkpatrick (1975)). However, the mathematical papers devoted to this general kind of model are rare: see Talagrand (2000a) on low temperature regime; Bardina et al. (2004) and Cadel et al. (2004) on high temperature regime; and Bovier et al. (2002) for some fluctuation results for the free energy.

We will denote by $\langle f\rangle$ the average of a function $f: \Sigma_{N} \rightarrow \mathbb{R}$ with respect to $G_{N}$, as well as the average of a function $f: \Sigma_{N}^{n} \rightarrow \mathbb{R}$ with respect to $G_{N}^{\otimes n}$, without mentioning the number $n$ of independent copies of the spin configurations, i.e.

$$
\langle f\rangle=\frac{1}{Z_{N}^{n}} \sum_{\left(\sigma^{1}, \ldots, \sigma^{n}\right) \in \Sigma_{N}^{n}} f\left(\sigma^{1}, \ldots, \sigma^{n}\right) \exp \left(-\sum_{l \leq n} H_{N, \beta, h}\left(\sigma^{l}\right)\right) .
$$

We write $\nu(f)=\mathbf{E}\langle f\rangle$, where $\mathbf{E}$ denotes expectation with respect to the randomness of the Hamiltonian.

The following assumption on $\beta$ determines our high temperature region:

(H) The parameter $\beta>0$ is smaller than a constant $\beta_{p}$ defined by

$$
8 p^{2} \beta_{p}^{2} \exp \left(16 \beta_{p}^{2} p\right)=\frac{1}{2} \text {. }
$$

In statistical mechanics, Gibbs' measure represents the probability of observing a configuration $\sigma$ after the system has reached equilibrium with an infinite heat bath at temperature $1 / \beta$. For this reason, $\beta$ small means high temperature.

Our aim is to prove the following theorem:

TheOrem 1.1. Assume $(\mathrm{H})$. Then, given a positive integer $m$, there exist independent standard Gaussian random variables $z_{1}, \ldots, z_{m}$ such that

$$
\mathbf{E} \sum_{i=1}^{m}\left[\left\langle\sigma_{i}\right\rangle-\tanh \left(\beta\left(\frac{p}{2}\right)^{1 / 2} q^{(p-1) / 2} z_{i}+h\right)\right]^{2} \leq \frac{C(m, h)}{N} .
$$

Here the constant $q=q_{p}$ is the unique solution of

$$
q=\mathbf{E}\left[\tanh ^{2}\left(\beta\left(\frac{p}{2}\right)^{1 / 2} q^{(p-1) / 2} Y+h\right)\right],
$$

where $Y$ stands for a standard Gaussian random variable. 
The constant $q=q_{p}$ is directly connected with the behavior of the overlap of two configurations

$$
R_{1,2}=\frac{1}{N} \sum_{i=1}^{N} \sigma_{i}^{1} \sigma_{i}^{2},
$$

and with the Hamming distance

$$
d\left(\sigma^{1}, \sigma^{2}\right)=\operatorname{card}\left\{i \leq N ; \sigma_{i}^{1}=\sigma_{i}^{2}\right\} .
$$

More specifically, for $\beta$ small enough, $R_{1,2}$ will self average into $q$ (see Proposition 2.1) and the knowledge of behavior of the overlap gives us information on this well-known distance by means of the equality

$$
d\left(\sigma^{1}, \sigma^{2}\right)=\frac{N}{2}\left(1-R_{1,2}\right) .
$$

For more information about the parameters $\beta_{p}$ and $q_{p}$ we refer the reader to Bardina et al. (2004).

As a consequence of Theorem 1.1, we have the following result:

Corollary 1.2. Assume $(\mathrm{H})$. Then the mean of a spin (with respect to the randomness of the configuration space) converges in law to an explicitly given random variable, namely

$$
\left\langle\sigma_{i}\right\rangle \underset{N \rightarrow \infty}{\stackrel{\mathcal{L}}{\longrightarrow}} \tanh \left(\beta\left(\frac{p}{2}\right)^{1 / 2} q^{(p-1) / 2} z_{i}+h\right) .
$$

Moreover, $\left\langle\sigma_{1}\right\rangle, \ldots,\left\langle\sigma_{n}\right\rangle$ are asymptotically independent.

In order to prove Theorem 1.1 we need the following important intermediate result.

Proposition 1.3. Given $\beta \leq \beta_{p}$, there exists a standard Gaussian random variable $z$ such that

$$
\mathbf{E}\left[\left\langle\sigma_{N}\right\rangle-\tanh \left(\beta\left(\frac{p}{2}\right)^{1 / 2} q^{(p-1) / 2} z+h\right)\right]^{2} \leq \frac{C(h)}{N},
$$

where $z$ depends only on $\left\{g_{J}: J \in A_{N}^{p}\right\}$ but is probabilistically independent of $\left\{g_{J}: J \in A_{N-1}^{p}\right\}$, with

$$
A_{N-1}^{p}=\left\{\left(i_{1}, \ldots, i_{p}\right) \in \mathbb{N}^{p}: 1 \leq i_{1}<\cdots<i_{p} \leq N-1\right\} .
$$

The paper is organized as follows: some preliminary results on the cavity method for our model are given in Section 2; Section 3 contains some intermediate results (Lemma 3.1) for the proof of Theorem 1.1, and the definition of the Gaussian path which will be used later on; the proofs of Lemma 3.1, Proposition 1.3 and Theorem 1.1 are given in Sections 4, 5 and 6, respectively. In the following, the size of a given finite set $D$ will be denoted by $|D|$. Let $C$ denote a constant which may vary from line to line. 
2. The cavity method. This method allows us, in some sense, to measure the difference between our original system and a system where the last spin is independent of the others. The cavity method for our model is already described in Bardina et al. (2004, Section 2.3), and it is given here only for the convenience of the reader.

For $\beta>0$, we define $\beta_{-}$that plays the role of $\beta$ in the new reduced system:

$$
\beta_{-}=\left(\frac{N-1}{N}\right)^{(p-1) / 2} \beta .
$$

Set

$$
Q_{N}^{p}=\left\{J=\left(i_{1}, \ldots, i_{p-1}, N\right) \in \mathbb{N}^{p} ; 1 \leq i_{1}<\cdots<i_{p-1} \leq N-1\right\},
$$

and recall that

$$
A_{N}^{p}=\left\{\left(i_{1}, \ldots, i_{p}\right) \in \mathbb{N}^{p} ; 1 \leq i_{1}<\cdots<i_{p} \leq N\right\} .
$$

Lemmas A.2 and A.4 in Bardina et al. (2004) prove that

$$
\begin{aligned}
& \left|A_{N}^{p}\right|=\left(\begin{array}{c}
N \\
p
\end{array}\right)=\frac{N^{p}}{p !}+P_{p-1}(N), \\
& \left|Q_{N}^{p}\right|=\left(\begin{array}{c}
N-1 \\
p-1
\end{array}\right)=\frac{N^{p-1}}{(p-1) !}+P_{p-2}(N),
\end{aligned}
$$

where $P_{m}(N)$ denotes some polynomial of degree $m$ in $N$. Moreover, as a consequence of Lemma A.4 in Bardina et al. (2004), it is not difficult to prove another deterministic result about the size of $Q_{N}^{p}$ :

$$
\left|u_{N}^{2}\right| Q_{N}^{p}\left|q^{p-1}-\frac{p}{2} q^{p-1}\right| \leq \frac{C}{N}
$$

for some positive constant $C$.

We use the following notation: $\varrho=\left(\sigma_{1}, \ldots, \sigma_{N-1}\right)$ is a configuration of $\Sigma_{N-1}, \eta_{J}=\sigma_{i_{1}} \cdots \sigma_{i_{p-1}}$ for $J \in Q_{N}^{p}$, and $\varepsilon=\sigma_{N}$. The basic idea of the cavity method is to regroup the Hamiltonian as follows:

$$
-H_{N, \beta, h}(\sigma)=-H_{N-1, \beta_{-}, h}(\varrho)+\varepsilon[g(\varrho)+h],
$$

where

$$
\begin{aligned}
-H_{N-1, \beta_{-}, h}(\varrho) & =\beta_{-} u_{N-1} \sum_{\left(i_{1}, \ldots, i_{p}\right) \in A_{N-1}^{p}} g_{i_{1}, \ldots, i_{p}} \sigma_{i_{1}} \ldots \sigma_{i_{p}}+h \sum_{i=1}^{N-1} \sigma_{i}, \\
g(\varrho) & =\beta u_{N} \sum_{J \in Q_{N}^{p}} g_{J} \eta_{J} .
\end{aligned}
$$

Let $\langle\cdot\rangle_{-}$denote the average with respect to Gibbs measure on $\Sigma_{N-1}$ relative to the reduced Hamiltonian $H_{N-1, \beta_{-}, h}$. In the spin glass theory, the cavity method becomes a powerful tool through the construction of a continuous 
path from the original configuration to a configuration where the last spin is independent of the others.

Set, for $t \in[0,1]$ and the constant $q \in[0,1]$ defined in (1.3),

$$
g_{t}(\varrho)=t^{1 / 2} g(\varrho)+\beta u_{N} q^{(p-1) / 2}(1-t)^{1 / 2} \sum_{J \in Q_{N}^{p}} z_{J},
$$

where $\left\{z_{J} ; J \in Q_{N}^{p}\right\}$ is a family of independent standard Gaussian random variables, also independent of all the disorders $g$.

For $n \geq 1$ and $n$ independent copies of an $N$-spin configuration $\sigma^{1}, \ldots, \sigma^{n}$, we write

$$
\begin{aligned}
\mathcal{E}_{n, t} & =\exp \left\{\sum_{l=1}^{n} \varepsilon^{l}\left[g_{t}\left(\varrho^{l}\right)+h\right]\right\}, \\
Z_{t} & =\left\langle\mathbf{A v} \mathcal{E}_{1, t}\right\rangle_{-}=\left\langle\cosh \left[g_{t}(\varrho)+h\right]\right\rangle_{-},
\end{aligned}
$$

where $\varepsilon^{l}=\sigma_{N}^{l}$ and $\mathbf{A v}$ means the average over $\left\{\varepsilon^{l} ; l=1, \ldots, n\right\}$. For $f: \Sigma_{N}^{n} \rightarrow \mathbb{R}$, we can define

$$
\langle f\rangle_{t}=\frac{\left\langle\mathbf{A v} f \mathcal{E}_{n, t}\right\rangle_{-}}{Z_{t}^{n}}, \quad \nu_{t}(f)=\mathbf{E}\langle f\rangle_{t} .
$$

Note that $\nu(f)=\nu_{1}(f)$.

The idea is that $\nu_{0}(f)$ (or a slight modification of it) should be simpler to compute than $\nu_{1}(f)$ in some interesting cases of functions $f$. On the other hand, we will relate these two quantities by means of

$$
\nu_{1}(f)-\nu_{0}(f)=\int_{0}^{1} \nu_{t}^{\prime}(f) d t .
$$

Let us summarize some results proved in Bardina et al. (2004) that will be useful in our proofs.

- For $t \in[0,1]$ and $f: \Sigma_{N}^{n} \rightarrow \mathbb{R}$, we have

$$
\begin{aligned}
\nu_{t}^{\prime}(f)= & \beta^{2} u_{N}^{2} \sum_{J \in Q_{N}^{p}}\left[\nu_{t}\left(f \sum_{1 \leq l<l^{\prime} \leq n}\left(\eta_{J}^{l} \eta_{J}^{l^{\prime}}-q^{p-1}\right) \varepsilon^{l} \varepsilon^{l^{\prime}}\right)\right. \\
& -n \nu_{t}\left(f \sum_{l=1}^{n}\left(\eta_{J}^{l} \eta_{J}^{n+1}-q^{p-1}\right) \varepsilon^{l} \varepsilon^{n+1}\right) \\
& \left.+\frac{n(n+1)}{2} \nu_{t}\left(f\left(\eta_{J}^{n+1} \eta_{J}^{n+2}-q^{p-1}\right) \varepsilon^{n+1} \varepsilon^{n+2}\right)\right] .
\end{aligned}
$$

- If $\tau_{1}, \tau_{2}>0$ are such that $1 / \tau_{1}+1 / \tau_{2}=1$, then, for any $t \in[0,1]$,

$$
\left|\nu_{t}\left(f_{1} f_{2}\right)\right| \leq \nu_{t}\left(\left|f_{1}\right|^{\tau_{1}}\right)^{1 / \tau_{1}} \nu_{t}\left(\left|f_{2}\right|^{\tau_{2}}\right)^{1 / \tau_{2}} .
$$


Proposition 2.1. Assume that $\beta$ satisfies $(\mathrm{H})$. Then, for $q \in[0,1]$ defined in (1.3) and for any $l \geq 1$,

$$
\begin{aligned}
\nu\left(\left(R_{1,2}-q\right)^{2 l}\right) & =\mathbf{E}\left\langle\left(R_{1,2}-q\right)^{2 l}\right\rangle \leq\left(\frac{C l}{N}\right)^{l}, \\
\left|\nu\left(R_{1,2}^{l}-q^{l}\right)\right| & \leq \frac{C(l)}{N},
\end{aligned}
$$

where $R_{1,2}$ has been defined in (1.4); and, for a function $f$ on $\Sigma_{N}^{n}$,

$$
\begin{aligned}
& \left|\nu(f)-\nu_{0}(f)\right| \leq \frac{C}{N^{1 / 2}} \nu^{1 / 2}\left(f^{2}\right), \\
& \left|\nu(f)-\nu_{0}(f)-\nu_{0}^{\prime}(f)\right| \leq \frac{C}{N} \nu^{1 / 2}\left(f^{2}\right) .
\end{aligned}
$$

Proof. See Proposition 3.2, Corollary 3.10 and Corollary 3.8 in Bardina et al. (2004).

3. Continuous path. The first and crucial step in the proof of Proposition 1.3 is the verification of the following two facts:

1. The average of $\sigma_{N}$ with respect to the Hamiltonian $H_{N, \beta, h}$ behaves asymptotically as the hyperbolic tangent of a quantity depending on $\left\{g_{J} ; J \in A_{N}^{p}\right\}$ but probabilistically independent of $\left\{g_{J} ; J \in A_{N-1}^{p}\right\}$.

2. The average of $\sigma_{1}$ with respect to the Hamiltonian $H_{N, \beta, h}$ behaves asymptotically as the average of the same spin $\sigma_{1}$ but only with respect to the Hamiltonian $H_{N-1, \beta_{-}, h}$.

These two facts can be deduced from the following lemma.

Lemma 3.1. Assume that $\beta$ satisfies $(\mathrm{H})$. Then, for $a \in\{0,1\}$,

$$
\Delta:=\mathbf{E}\left[\left\langle\sigma_{1}^{a} \varepsilon^{1-a}\right\rangle-\left\langle\sigma_{1}^{a}\right\rangle_{-} \tanh ^{1-a}\left(\beta u_{N} \sum_{J \in Q_{N}^{p}} g_{J}\left\langle\eta_{J}\right\rangle_{-}+h\right)\right]^{2} \leq \frac{C}{N} .
$$

We start by giving the definition of the Gaussian path we will use: let

$$
\widetilde{g}(c)=\beta u_{N} \sum_{J \in Q_{N}^{p}} g_{J}\left\langle\eta_{J}\right\rangle_{-},
$$

and, for $t \in[0,1]$,

$$
\widetilde{g}_{t}(c)=t^{1 / 2} \widetilde{g}(c)+\beta u_{N} q^{(p-1) / 2}(1-t)^{1 / 2} \sum_{J \in Q_{N}^{p}} z_{J},
$$


where $\left\{z_{J} ; J \in Q_{N}^{p}\right\}$ is as in (2.2). As in (2.3) and (2.4), for $n \geq 1$ and $n$ independent copies of an $N$-spin configuration $\sigma^{1}, \ldots, \sigma^{n}$, we can define

$$
\begin{aligned}
\widetilde{\mathcal{E}}_{n, t} & =\exp \left\{\sum_{l=1}^{n} \varepsilon^{l}\left[\widetilde{g}_{t}(c)+h\right]\right\}, \\
\widetilde{Z}_{t} & =\left\langle\operatorname{Av} \mathbf{v} \widetilde{\mathcal{E}}_{1, t}\right\rangle_{-}=\left\langle\cosh \left[\widetilde{g}_{t}(c)+h\right]\right\rangle_{-} .
\end{aligned}
$$

Then, for $t \in[0,1]$, we consider the function

$$
\Theta(t)=\mathbf{E}\left[(\Phi(t)-\Psi(t))^{2}\right],
$$

where, for $a \in\{0,1\}$,

$$
\begin{aligned}
& \Phi(t):=\left\langle\sigma_{1}^{a} \varepsilon^{1-a}\right\rangle_{t}=\frac{\left\langle\mathbf{A v} \sigma_{1}^{a} \varepsilon^{1-a} \mathcal{E}_{1, t}\right\rangle_{-}}{Z_{t}}, \\
& \Psi(t):=\frac{\left\langle\mathbf{A v} \sigma_{1}^{a} \varepsilon^{1-a} \widetilde{\mathcal{E}}_{1, t}\right\rangle_{-}}{\widetilde{Z}_{t}}=\left\langle\sigma_{1}^{a}\right\rangle_{-} \tanh ^{1-a}\left[\widetilde{g}_{t}(c)+h\right] .
\end{aligned}
$$

We can decompose $\Theta$ into three terms

$$
\Theta(t)=\Theta_{1}(t)+\Theta_{2}(t)+\Theta_{3}(t),
$$

with

$$
\begin{aligned}
\Theta_{1}(t) & =\mathbf{E}\left[\Phi(t)^{2}\right], \\
\Theta_{2}(t) & =\mathbf{E}\left[\Psi(t)^{2}\right], \\
\Theta_{3}(t) & =-2 \mathbf{E}[\Phi(t) \Psi(t)] .
\end{aligned}
$$

Since it is easy to check that $\Phi(0)=\Psi(0)$, it follows that $\Delta$, defined in (3.1), satisfies

$$
\Delta=|\Theta(1)|=|\Theta(1)-\Theta(0)| \leq \sum_{j=1}^{3}\left[\left|\Theta_{j}(1)-\Theta_{j}(0)-\Theta_{j}^{\prime}(0)\right|+\left|\Theta_{j}^{\prime}(0)\right|\right] .
$$

Thus, (3.1) in Lemma 3.1 will be achieved as soon as we can show that

$$
\left|\Theta_{j}(1)-\Theta_{j}(0)-\Theta_{j}^{\prime}(0)\right| \vee\left|\Theta_{j}^{\prime}(0)\right| \leq C / N \quad \text { for any } j=1,2,3 \text {. }
$$

\section{Proof of Lemma 3.1}

4.1. Study of $\Theta_{1}$. Using two replicas of $\sigma$, we obtain

$$
\Theta_{1}(t)=\mathbf{E}\left[\Phi(t)^{2}\right]=\mathbf{E}\left\langle\sigma_{1}^{a} \varepsilon^{1-a}\right\rangle_{t}^{2}=\nu_{t}\left(\left(\sigma_{1}^{1} \sigma_{1}^{2}\right)^{a}\left(\varepsilon^{1} \varepsilon^{2}\right)^{1-a}\right),
$$

where the measure $\nu_{t}$ is defined in Section 2; recall that $a \in\{0,1\}$.

First of all, since $\left|\left(\sigma_{1}^{1} \sigma_{1}^{2}\right)^{a}\left(\varepsilon^{1} \varepsilon^{2}\right)^{1-a}\right| \leq 1$, by (2.11) in Proposition 2.1, we have

$$
\left|\Theta_{1}(1)-\Theta_{1}(0)-\Theta_{1}^{\prime}(0)\right| \leq C / N .
$$

Thus, if we check that $\left|\Theta_{1}^{\prime}(0)\right| \leq C / N$, we will have proved (3.4) when $j=1$ and concluded the study of $\Theta_{1}$. From (2.6), the symmetry and independence 
yield

$$
\begin{aligned}
\Theta_{1}^{\prime}(0)= & \nu_{0}^{\prime}\left(\left(\sigma_{1}^{1} \sigma_{1}^{2}\right)^{a}\left(\varepsilon^{1} \varepsilon^{2}\right)^{1-a}\right) \\
= & \beta^{2} u_{N}^{2} \sum_{J \in Q_{N}^{p}}\left[\nu_{0}\left(\left(\sigma_{1}^{1} \sigma_{1}^{2}\right)^{a}\left(\eta_{J}^{1} \eta_{J}^{2}-q^{p-1}\right)\right) \nu_{0}\left(\left(\varepsilon^{1} \varepsilon^{2}\right)^{2-a}\right)\right. \\
& -4 \nu_{0}\left(\left(\sigma_{1}^{1} \sigma_{1}^{2}\right)^{a}\left(\eta_{J}^{1} \eta_{J}^{3}-q^{p-1}\right)\right) \nu_{0}\left(\left(\varepsilon^{1}\right)^{2-a}\left(\varepsilon^{2}\right)^{1-a} \varepsilon^{3}\right) \\
& \left.+3 \nu_{0}\left(\left(\sigma_{1}^{1} \sigma_{1}^{2}\right)^{a}\left(\eta_{J}^{3} \eta_{J}^{4}-q^{p-1}\right)\right) \nu_{0}\left(\left(\varepsilon^{1} \varepsilon^{2}\right)^{1-a} \varepsilon^{3} \varepsilon^{4}\right)\right] .
\end{aligned}
$$

So, in order to bound $\left|\Theta_{1}^{\prime}(0)\right|$, since $|\varepsilon| \leq 1$, we only need to check that, for any couple $(i, j) \in\{(1,2),(1,3),(3,4)\}$,

$$
\Upsilon:=\left|\beta^{2} u_{N}^{2} \sum_{J \in Q_{N}^{p}} \nu_{0}\left(\left(\sigma_{1}^{1} \sigma_{1}^{2}\right)^{a}\left(\eta_{J}^{i} \eta_{J}^{j}-q^{p-1}\right)\right)\right| \leq C / N .
$$

The quantity $\Upsilon$ can be bounded by three terms as follows:

$$
\Upsilon \leq \beta^{2}\left[\Upsilon_{1}+\Upsilon_{2}+\Upsilon_{3}\right]
$$

with

$$
\begin{aligned}
& \Upsilon_{1}=\left|u_{N}^{2} \sum_{J \in Q_{N}^{p}} \nu_{0}\left(\left(\sigma_{1}^{1} \sigma_{1}^{2}\right)^{a} \eta_{J}^{i} \eta_{J}^{j}\right)-\frac{p}{2} \nu_{0}\left(\left(\sigma_{1}^{1} \sigma_{1}^{2}\right)^{a} R_{i, j}^{p-1}\right)\right|, \\
& \Upsilon_{2}=\frac{p}{2}\left|\nu_{0}\left(\left(\sigma_{1}^{1} \sigma_{1}^{2}\right)^{a} R_{i, j}^{p-1}\right)-\nu_{0}\left(\left(\sigma_{1}^{1} \sigma_{1}^{2}\right)^{a} q^{p-1}\right)\right|, \\
& \Upsilon_{3}=\left|\frac{p}{2} \nu_{0}\left(\left(\sigma_{1}^{1} \sigma_{1}^{2}\right)^{a} q^{p-1}\right)-u_{N}^{2} \sum_{J \in Q_{N}^{p}} \nu_{0}\left(\left(\sigma_{1}^{1} \sigma_{1}^{2}\right)^{a} q^{p-1}\right)\right| .
\end{aligned}
$$

Recall that $R_{1,2}$ has been defined in (1.4). On the one hand, Lemma 5.11 in Talagrand (2000a) gives

$$
\left|u_{N}^{2} \sum_{J \in Q_{N}^{p}} \eta_{J}^{i} \eta_{J}^{j}-\frac{p}{2} R_{i, j}^{p-1}\right| \leq C / N,
$$

which together with the estimate (2.1) implies

$$
\beta^{2}\left(\Upsilon_{1}+\Upsilon_{3}\right) \leq C / N
$$

On the other hand, we have

$$
\Upsilon_{2}=\frac{p}{2}\left|\nu_{0}\left(\left(\sigma_{1}^{1} \sigma_{1}^{2}\right)^{a}\left(R_{i, j}^{p-1}-q^{p-1}\right)\right)\right| \leq \frac{p}{2}\left[\Upsilon_{2,1}+\Upsilon_{2,2}\right],
$$

where

$$
\begin{aligned}
& \Upsilon_{2,1}=\left|\nu_{0}\left(\left(\sigma_{1}^{1} \sigma_{1}^{2}\right)^{a}\left(R_{i, j}^{p-1}-q^{p-1}\right)\right)-\nu\left(\left(\sigma_{1}^{1} \sigma_{1}^{2}\right)^{a}\left(R_{i, j}^{p-1}-q^{p-1}\right)\right)\right|, \\
& \Upsilon_{2,2}=\left|\nu\left(\left(\sigma_{1}^{1} \sigma_{1}^{2}\right)^{a}\left(R_{i, j}^{p-1}-q^{p-1}\right)\right)\right| .
\end{aligned}
$$

Applying the estimates (2.10) and (2.8) for $l=1$, and using the fact that 
$\left|\left(\sigma_{1}^{1} \sigma_{1}^{2}\right)^{a}\right| \vee\left|R_{i, j}\right| \vee q \leq 1$, we obtain

$$
\begin{aligned}
\Upsilon_{2,1} & \leq \frac{C}{N^{1 / 2}}\left[\nu\left(\left(R_{i, j}^{p-1}-q^{p-1}\right)^{2}\right)\right]^{1 / 2} \\
& \leq \frac{C}{N^{1 / 2}}\left[\nu\left(\left(R_{i, j}-q\right)^{2}\right)\right]^{1 / 2} \leq \frac{C}{N} .
\end{aligned}
$$

Using the symmetry, the Cauchy-Schwarz inequality (2.7) and Proposition 2.1 (in particular, the bounds (2.8) and (2.9)), we get

$$
\begin{aligned}
\Upsilon_{2,2} & =\left|\nu\left(R_{1,2}^{a}\left(R_{i, j}^{p-1}-q^{p-1}\right)\right)\right| \\
& \leq\left|\nu\left(\left(R_{1,2}-q\right)^{a}\left(R_{i, j}^{p-1}-q^{p-1}\right)\right)\right|+q^{a}\left|\nu\left(R_{i, j}^{p-1}-q^{p-1}\right)\right| \\
& \leq C / N .
\end{aligned}
$$

Putting together (4.3)-(4.6) provides (4.1), which concludes the study of $\Theta_{1}$.

4.2. Study of $\Theta_{2}$. For $t \in[0,1]$ and $f: \Sigma_{N}^{n} \rightarrow \mathbb{R}$, consider the new measure $\widetilde{\nu}_{t}$ defined by

$$
\widetilde{\nu}_{t}(f)=\mathbf{E}\left(\frac{\left\langle\mathbf{A v} f \widetilde{\mathcal{E}}_{n, t}\right\rangle_{-}}{\widetilde{Z}_{t}^{n}}\right)
$$

where $\widetilde{\mathcal{E}}_{n, t}$ and $\widetilde{Z}_{t}$ are given in (3.2) and (3.3), respectively.

Working as in Proposition 2.1 of Bardina et al. (2004), we can express the derivative of this new measure as

$$
\begin{aligned}
\widetilde{\nu}_{t}^{\prime}(f)= & \beta^{2} u_{N}^{2} \sum_{J \in Q_{N}^{p}}\left[\widetilde{\nu}_{t}\left(f\left(\left\langle\eta_{J}\right\rangle_{-}^{2}-q^{p-1}\right) \sum_{1 \leq l<l^{\prime} \leq n} \varepsilon^{l} \varepsilon^{l^{\prime}}\right)\right. \\
& -n \widetilde{\nu}_{t}\left(f\left(\left\langle\eta_{J}\right\rangle_{-}^{2}-q^{p-1}\right) \sum_{l=1}^{n} \varepsilon^{l} \varepsilon^{n+1}\right) \\
& \left.+\frac{n(n+1)}{2} \widetilde{\nu}_{t}\left(f\left(\left\langle\eta_{J}\right\rangle_{-}^{2}-q^{p-1}\right) \varepsilon^{n+1} \varepsilon^{n+2}\right)\right] .
\end{aligned}
$$

First of all, taking two replicas of $\sigma$ allows us to write $\Theta_{2}$, for $a \in\{0,1\}$, as

$$
\Theta_{2}(t)=\mathbf{E}\left[\Psi(t)^{2}\right]=\widetilde{\nu}_{t}\left(\left(\sigma_{1}^{1} \sigma_{1}^{2}\right)^{a}\left(\varepsilon^{1} \varepsilon^{2}\right)^{1-a}\right) .
$$

Then, in order to bound $\left|\Theta_{2}^{\prime}(0)\right|$, we will use (4.7) with $f=\left(\sigma_{1}^{1} \sigma_{1}^{2}\right)^{a}\left(\varepsilon^{1} \varepsilon^{2}\right)^{1-a}$. So, by symmetry and independence, using the fact that $\left|\varepsilon^{i} \varepsilon^{j}\right| \leq 1$, the 
definition of $\widetilde{\nu_{t}}$ for $t=0$, and taking new replicas of $\sigma$, we obtain

$$
\begin{aligned}
\left|\Theta_{2}^{\prime}(0)\right| & \leq 8\left|\beta^{2} u_{N}^{2} \sum_{J \in Q_{N}^{p}} \widetilde{\nu}_{0}\left(\left(\sigma_{1}^{1} \sigma_{1}^{2}\right)^{a}\left(\left\langle\eta_{J}\right\rangle_{-}^{2}-q^{p-1}\right)\right)\right| \\
& =8 \mid \beta^{2} u_{N}^{2} \sum_{J \in Q_{N}^{p}} \mathbf{E}\left\langle\left(\sigma_{1}^{1} \sigma_{1}^{2}\right)^{a}\left(\left\langle\eta_{J}^{3} \eta_{J}^{4}-q^{p-1}\right\rangle_{-}\right\rangle_{-}\right| \\
& =8\left|\beta^{2} u_{N}^{2} \sum_{J \in Q_{N}^{p}} \nu_{0}\left(\left(\sigma_{1}^{1} \sigma_{1}^{2}\right)^{a}\left(\eta_{J}^{3} \eta_{J}^{4}-q^{p-1}\right)\right)\right| .
\end{aligned}
$$

We now proceed as for the study of (4.1) to prove that

$$
\left|\Theta_{2}^{\prime}(0)\right| \leq C / N \text {. }
$$

It remains to analyze the other term of (3.4) for $j=2$. Taylor expansion applied to (4.8) yields

$$
\left|\Theta_{2}(1)-\Theta_{2}(0)-\Theta_{2}^{\prime}(0)\right|=\left|\widetilde{\nu}_{1}(f)-\widetilde{\nu}_{0}(f)-\widetilde{\nu}_{0}^{\prime}(f)\right| \leq \frac{1}{2} \int_{0}^{1}\left|\widetilde{\nu}_{t}^{\prime \prime}(f)\right| d t
$$

for $f=\left(\sigma_{1}^{1} \sigma_{1}^{2}\right)^{a}\left(\varepsilon^{1} \varepsilon^{2}\right)^{1-a}$. Bounding accurately the derivative of (4.7) we obtain

$$
\left|\widetilde{\nu}_{t}^{\prime \prime}(f)\right| \leq C \beta^{4} u_{N}^{4}\left|\sum_{J_{1}, J_{2} \in Q_{N}^{p}} \widetilde{\nu}_{t}\left(f\left[\left\langle\eta_{J_{1}}\right\rangle_{-}^{2}-q^{p-1}\right]\left[\left\langle\eta_{J_{2}}\right\rangle_{-}^{2}-q^{p-1}\right] \widehat{\varepsilon}\right)\right|
$$

with $\widehat{\varepsilon}=\varepsilon^{i} \varepsilon^{j} \varepsilon^{i^{\prime}} \varepsilon^{j^{\prime}}$. Then, considering different replicas of $\sigma$, using the fact that $|f \widehat{\varepsilon}| \vee\left|R_{1,2}\right| \vee q \leq 1$ and applying (4.2) and (2.1) (as in (4.3) for $\Upsilon$ ), we get

$$
\begin{aligned}
\left|\nu_{t}^{\prime \prime}(f)\right| & \leq C \beta^{4}\left|\widetilde{\nu}_{t}\left(f \widehat{\varepsilon}\left\langle u_{N}^{2} \sum_{J_{\in} Q_{N}^{p}}\left[\eta_{J}^{1} \eta_{J}^{2}-q^{p-1}\right]\right\rangle_{-}^{2}\right)\right| \\
& \leq C \beta^{4} \mathbf{E}\left(\frac{1}{\widetilde{Z}_{t}^{2}}\left\langle\mathbf{A v}\left\langle u_{N}^{2} \sum_{J_{\in} Q_{N}^{p}}\left[\eta_{J}^{1} \eta_{J}^{2}-q^{p-1}\right]\right\rangle_{-}^{2} \widetilde{\mathcal{E}}_{2, t}\right\rangle_{-}\right) \\
& =C \beta^{4} \mathbf{E}\left(\left\langle u_{N}^{2} \sum_{J_{\in} Q_{N}^{p}}\left[\eta_{J}^{1} \eta_{J}^{2}-q^{p-1}\right]\right\rangle_{-}^{2}\right) \\
& \leq C \beta^{4} \nu_{0}\left(\left|\left(R_{1,2}^{p-1}-q^{p-1}\right)\left(R_{3,4}^{p-1}-q^{p-1}\right)\right|\right)+C / N
\end{aligned}
$$

and now we proceed as in (4.4) for $\Upsilon_{2}$ to conclude that

$$
\left|\Theta_{2}(1)-\Theta_{2}(0)-\Theta_{2}^{\prime}(0)\right| \leq C / N
$$

This estimate together with (4.10) ends the study of $\Theta_{2}$. 
4.3. Study of $\Theta_{3}$. Here the term $\Theta_{3}$ is, in some sense, a mixture between $\Theta_{1}$ and $\Theta_{2}$. For $t \in[0,1]$ and

$$
f: \Sigma_{N}^{n} \times \Sigma_{N}^{\widetilde{n}} \rightarrow \mathbb{R}, \quad(\sigma, \widetilde{\sigma}) \mapsto f(\sigma, \widetilde{\sigma}),
$$

we define

$$
\widehat{\nu}_{t}(f)=\mathbf{E}\left(\frac{1}{Z_{t}^{n}(\sigma) \widetilde{Z_{t}^{\tilde{n}}}(\widetilde{\sigma})}\left\langle\widehat{\mathbf{A v}} f(\sigma, \widetilde{\sigma}) \mathcal{E}_{n, t}(\sigma) \widetilde{\mathcal{E}}_{\widetilde{n}, t}(\widetilde{\sigma})\right\rangle_{-}\right),
$$

where $\widehat{\mathbf{A v}}$ means the average over $\left\{\varepsilon^{l}, \widetilde{\varepsilon}^{\widetilde{l}} ; l=1, \ldots, n, \widetilde{l}=1, \ldots, \widetilde{n}\right\}$.

It is long and tedious but not difficult to deduce that the derivative of $\widehat{\nu_{t}}(f)$ is composed of three kinds of terms, namely

$$
\begin{aligned}
& \Xi_{1, t}(f)=\beta^{2} u_{N}^{2} \sum_{J_{\in} Q_{N}^{p}} \widehat{\nu}_{t}\left(f(\sigma, \widetilde{\sigma})\left[\eta_{J}^{l} \eta_{J}^{l^{\prime}}-q^{p-1}\right] \varepsilon^{l} \varepsilon^{l^{\prime}}\right), \\
& \Xi_{2, t}(f)=\beta^{2} u_{N}^{2} \sum_{J_{\in} Q_{N}^{p}} \widehat{\nu}_{t}\left(f(\sigma, \widetilde{\sigma})\left[\left\langle\widetilde{\eta}_{J}\right\rangle_{-}^{2}-q^{p-1}\right] \widetilde{\varepsilon}^{\widetilde{l}} \widetilde{\varepsilon}^{\widetilde{c}^{\prime}}\right), \\
& \Xi_{3, t}(f)=\beta^{2} u_{N}^{2} \sum_{J_{\in} Q_{N}^{p}} \widehat{\nu}_{t}\left(f(\sigma, \widetilde{\sigma})\left[\eta_{J}^{l}\left\langle\widetilde{\eta}_{J}\right\rangle_{-}-q^{p-1}\right] \varepsilon^{l} \widetilde{\varepsilon}^{\vec{l}}\right),
\end{aligned}
$$

where $l, l^{\prime} \in\{1, \ldots, n+2\}, \widetilde{l}, \widetilde{l^{\prime}} \in\{1, \ldots, \widetilde{n}+2\}$. As in the previous sections, we also have, for $a \in\{0,1\}$,

$$
\Theta_{3}(t)=-2 \mathbf{E}[\Phi(t) \Psi(t)]=-2 \widehat{\nu}_{t}\left(\left(\sigma_{1} \widetilde{\sigma}_{1}\right)^{a}(\varepsilon \widetilde{\varepsilon})^{1-a}\right) .
$$

In order to check that $\left|\Theta_{3}(0)\right| \leq C / N$, the cases $\Xi_{1,0}(f)$ and $\Xi_{2,0}(f)$ (with $\left.f=\left(\sigma_{1} \widetilde{\sigma}_{1}\right)^{a}(\varepsilon \widetilde{\varepsilon})^{1-a}\right)$ are handled as in the subsections devoted to $\Theta_{1}$ and $\Theta_{2}$, respectively. In the remaining case, by symmetry and independence we have

$$
\begin{aligned}
\left|\Xi_{3,0}(f)\right| & =\beta^{2} u_{N}^{2}\left|\sum_{J_{\in} Q_{N}^{p}} \widehat{\nu}_{0}\left(\left(\sigma_{1}^{1} \widetilde{\sigma}_{1}^{1}\right)^{a}\left(\varepsilon^{1} \widetilde{\varepsilon}^{1}\right)^{1-a}\left[\eta_{J}^{l}\left\langle\widetilde{\eta}_{J}\right\rangle_{-}-q^{p-1}\right] \varepsilon^{l} \widetilde{\varepsilon}^{\vec{l}}\right)\right| \\
& \leq \beta^{2} u_{N}^{2}\left|\sum_{J_{\in} Q_{N}^{p}} \widehat{\nu}_{0}\left(\left(\sigma_{1}^{1} \widetilde{\sigma}_{1}^{1}\right)^{a}\left[\eta_{J}^{l}\left\langle\widetilde{\eta}_{J}\right\rangle_{-}-q^{p-1}\right]\right)\right| \\
& =\beta^{2} u_{N}^{2}\left|\sum_{J_{\in} Q_{N}^{p}} \mathbf{E}\left\langle\left(\sigma_{1}^{1} \sigma_{1}^{2}\right)^{a}\left[\eta_{J}^{k} \eta_{J}^{3}-q^{p-1}\right]\right\rangle-\right| \\
& =\beta^{2} u_{N}^{2}\left|\sum_{J_{\in} Q_{N}^{p}} \nu_{0}\left(\left(\sigma_{1}^{1} \sigma_{1}^{2}\right)^{a}\left[\eta_{J}^{k} \eta_{J}^{3}-q^{p-1}\right]\right)\right|,
\end{aligned}
$$

where $k$ is equal to 1 or 4 . Now, since $\left|\Xi_{3,0}(f)\right|$ is bounded by the same type of factor as $\Upsilon$ in (4.1), we proceed as in the study of $\Upsilon$ in Section 4.1.

Finally, we can conclude that

$$
\left|\Theta_{3}(1)-\Theta_{3}(0)-\Theta_{3}^{\prime}(0)\right| \leq \frac{1}{2} \int_{0}^{1} \widehat{\nu}_{t}^{\prime \prime}(f) d t \quad \text { with } \quad f=\left(\sigma_{1} \widetilde{\sigma}_{1}\right)^{a}(\varepsilon \widetilde{\varepsilon})^{1-a} .
$$


Since the terms of this second derivative are of the same type as $\Theta_{1}$ or $\Theta_{2}$ or a mixture between $\Theta_{1}$ and $\Theta_{2}$, they can be dealt with as in Sections 4.1, 4.2 or as in (4.12).

\section{Proof of Proposition 1.3. Let}

$$
z=\frac{1}{\|c\|} \sum_{J \in Q_{N}^{p}} g_{J}\left\langle\eta_{J}\right\rangle_{-} \quad \text { with } \quad\|c\|^{2}=\sum_{J \in Q_{N}^{p}}\left\langle\eta_{J}\right\rangle_{-}^{2} .
$$

It will be observed later that this $z$ is the random variable appearing in Proposition 1.3. Let us start with an easy but curious property of $z$ that will be used in the proof of this proposition.

Lemma 5.1. The law of $z$ is standard Gaussian. This random variable depends only on $\left\{g_{J} ; J \in A_{N}^{p}\right\}$ but is independent of $\left\{g_{J} ; J \in A_{N-1}^{p}\right\}$.

Proof. Since $A_{N}^{p}=A_{N-1}^{p} \dot{\cup} Q_{N}^{p}$, it is obvious that $z$ depends on $\left\{g_{J} ; J \in A_{N}^{p}\right\}$. Moreover, conditionally upon $\left\{\left\langle\eta_{J}\right\rangle_{-} ; J \in Q_{N}^{p}\right\}$, the law of $(1 /\|c\|) g_{J}\left\langle\eta_{J}\right\rangle_{-}$is trivially centered Gaussian with variance $\left(1 /\|c\|^{2}\right)\left\langle\eta_{J}\right\rangle_{-}^{2}$. So, denoting by $E_{-}$the conditional expectation upon $\left\{\left\langle\eta_{J}\right\rangle_{-} ; J \in Q_{N}^{p}\right\}$, by conditional independence we can get

$$
\begin{aligned}
\mathbf{E}\left(e^{i v z}\right) & =\mathbf{E}\left[E_{-}\left(e^{i v z}\right)\right]=\mathbf{E}\left[E_{-}\left(\prod_{J \in Q_{N}^{p}} \exp \left\{\frac{1}{\|c\|} i v g_{J}\left\langle\eta_{J}\right\rangle_{-}\right\}\right)\right] \\
& =\mathbf{E}\left[\prod_{J \in Q_{N}^{p}} E_{-}\left(\exp \left\{\frac{1}{\|c\|} i v g_{J}\left\langle\eta_{J}\right\rangle_{-}\right\}\right)\right] \\
& =\mathbf{E}\left[\prod_{J \in Q_{N}^{p}} \exp \left\{-\frac{v^{2}\left\langle\eta_{J}\right\rangle_{-}^{2}}{2\|c\|^{2}}\right\}\right]=e^{-v^{2} / 2},
\end{aligned}
$$

which implies that $z$ is a standard Gaussian random variable. Finally, $z$ is independent of $\left\{g_{J} ; J \in A_{N-1}^{p}\right\}$ since we can check that $\mathbf{E}\left[z g_{\widetilde{J}}\right]=0$ for any $g_{\widetilde{J}}, \widetilde{J} \in A_{N-1}^{p}$.

Proof of Proposition 1.3. We want to show that

$$
\Lambda:=\mathbf{E}\left[\left\langle\sigma_{N}\right\rangle-\tanh \left(\beta\left(\frac{p}{2}\right)^{1 / 2} q^{(p-1) / 2} z+h\right)\right]^{2} \leq \frac{C(h)}{N},
$$

where $z$ is defined in (5.1).

We can write

$$
\Lambda \leq 2\left(\Lambda_{1}+\Lambda_{2}\right)
$$


with

$$
\begin{aligned}
& \Lambda_{1}=\mathbf{E}\left[\left\langle\sigma_{N}\right\rangle-\tanh (\widetilde{g}(c)+h)\right]^{2}, \\
& \Lambda_{2}=\mathbf{E}\left[\tanh \left(\beta\left(\frac{p}{2}\right)^{1 / 2} q^{(p-1) / 2} z+h\right)-\tanh (\widetilde{g}(c)+h)\right]^{2} .
\end{aligned}
$$

We only need to study $\Lambda_{2}$ because Lemma 3.1 for $a=0$ implies $\Lambda_{1} \leq C / N$. Using the inequality $|\tanh a-\tanh b| \leq|a-b|$, the definitions of $\widetilde{g}(c), z$ and $\|c\|$, and the conditional expectation $E_{-}$defined in Lemma 5.1, we obtain

$$
\begin{aligned}
\Lambda_{2} & \leq \beta^{2} \mathbf{E}\left[\left(\frac{p}{2}\right)^{1 / 2} q^{(p-1) / 2} z-u_{N} \sum_{J \in Q_{N}^{p}} g_{J}\left\langle\eta_{J}\right\rangle_{-}\right]^{2} \\
& =\beta^{2} \mathbf{E}\left[E_{-}\left\{\left(\frac{1}{\|c\|}\left(\frac{p}{2}\right)^{1 / 2} q^{(p-1) / 2}-u_{N}\right) \sum_{J \in Q_{N}^{p}} g_{J}\left\langle\eta_{J}\right\rangle_{-}\right\}^{2}\right] \\
& =\beta^{2} \mathbf{E}\left[\left(\frac{1}{\|c\|}\left(\frac{p}{2}\right)^{1 / 2} q^{(p-1) / 2}-u_{N}\right)^{2} \sum_{J \in Q_{N}^{p}}\left\langle\eta_{J}\right\rangle_{-}^{2}\right] \\
& =\beta^{2} \mathbf{E}\left(\left(\frac{p}{2}\right)^{1 / 2} q^{(p-1) / 2}-u_{N} \sqrt{\sum_{J \in Q_{N}^{p}}\left\langle\eta_{J}\right\rangle_{-}^{2}}\right)^{2} .
\end{aligned}
$$

When $h=0$, we have $q=0$, hence the result. Assume now that $h>0$. Then, since the lower bound of $q$ (solution of (1.3)) is uniform in $\beta \leq \beta_{p}$, by means of (2.1) we have

$$
\begin{aligned}
\Lambda_{2} & \leq \frac{2 \beta^{2}}{p q^{p-1}} \mathbf{E}\left(\frac{p}{2} q^{p-1}-u_{N}^{2} \sum_{J \in Q_{N}^{p}}\left\langle\eta_{J}\right\rangle_{-}^{2}\right)^{2} \\
& \leq \frac{2 \beta^{2} u_{N}^{4}}{p q^{p-1}} \mathbf{E}\left(\sum_{J \in Q_{N}^{p}}\left[q^{p-1}-\left\langle\eta_{J}\right\rangle_{-}^{2}\right]\right)^{2}+\frac{C}{N} .
\end{aligned}
$$

This last term can be bounded as in (4.11).

6. Proof of Theorem 1.1. A last result will be needed to be able to prove this theorem.

LEMMA 6.1. Let $q$ be the unique solution of (1.3) and $q_{-}$the unique solution of

$$
q_{-}=\mathbf{E}\left[\tanh ^{2}\left(\beta_{-}\left(\frac{p}{2}\right)^{1 / 2} q_{-}^{(p-1) / 2} Y+h\right)\right]
$$

with $\beta_{-}=((N-1) / N)^{(p-1) / 2} \beta$ and $Y$ as in (1.3). Then, if $\beta \leq \beta_{p}$, we have

$$
\left|q-q_{-}\right| \leq C / N \text {. }
$$


Proof. Lemma 2.4.15 in Talagrand (2000b) proves the case $p=2$. Assume $p \geq 3$. For $s>0$, set $\lambda(s)=\mathbf{E} \tanh ^{2}\left(X_{s}+h\right)$, where $X_{s}$ is a centered Gaussian random variable with variance $s^{2}$. It is not difficult to check that $\left|\lambda^{\prime}(s)\right| \leq C$. Then, by using the mean value theorem and the fact that $\left|q \vee q_{-}\right| \leq 1$, we obtain

$$
\begin{aligned}
\left|q-q_{-}\right| & =\left|\lambda\left(\beta\left(\frac{p}{2}\right)^{1 / 2} q^{(p-1) / 2}\right)-\lambda\left(\beta_{-}\left(\frac{p}{2}\right)^{1 / 2} q_{-}^{(p-1) / 2}\right)\right| \\
& \leq C\left|\beta q^{(p-1) / 2}-\beta_{-} q_{-}^{(p-1) / 2}\right| \leq C\left[\left|\beta-\beta_{-}\right|+\beta\left|q^{(p-1) / 2}-q_{-}^{(p-1) / 2}\right|\right] \\
& \leq C / N+C \beta\left|q-q_{-}\right| .
\end{aligned}
$$

Taking $\beta$ small enough, we have

$$
\left|q-q_{-}\right| \leq \frac{C}{(1-C \beta) N} \leq \frac{C}{2 N}
$$

Proof of Theorem 1.1. We argue by induction. We assume that the random variables $\left\{z_{1}, \ldots, z_{m}\right\}$ depend on $\left\{g_{i_{1}, \ldots, i_{p}} ;\left(i_{1}, \ldots, i_{p}\right) \in A_{N}^{p}\right\}$ as part of the induction hypothesis. The case $m=1$ is a consequence of the symmetry applied to Proposition 1.3.

We now assume that Theorem 1.1 is true for $m$ and we will check it for $m+1$. In order to show the independence of the random variables $\left\{z_{1}, \ldots, z_{m}, z_{m+1}\right\}$ of Theorem 1.1, we need to apply the induction hypothesis to the $N-1$-spin system with Hamiltonian $H_{N-1, \beta_{-}, h}$. First of all, we make the following decomposition:

$$
\mathbf{E} \sum_{i=1}^{m+1}\left[\left\langle\sigma_{i}\right\rangle-\tanh \left(\beta\left(\frac{p}{2}\right)^{1 / 2} q^{(p-1) / 2} z_{i}+h\right)\right]^{2} \leq C \sum_{j=1}^{4} \Gamma_{j}
$$

with

$$
\begin{aligned}
\Gamma_{1}= & \mathbf{E} \sum_{i=1}^{m}\left[\left\langle\sigma_{i}\right\rangle-\left\langle\sigma_{i}\right\rangle_{-}\right]^{2}, \\
\Gamma_{2}= & \mathbf{E} \sum_{i=1}^{m}\left[\left\langle\sigma_{i}\right\rangle_{-}-\tanh \left(\beta_{-}\left(\frac{p}{2}\right)^{1 / 2} q_{-}^{(p-1) / 2} z_{i}+h\right)\right]^{2}, \\
\Gamma_{3}= & \mathbf{E} \sum_{i=1}^{m}\left[\tanh \left(\beta_{-}\left(\frac{p}{2}\right)^{1 / 2} q_{-}^{(p-1) / 2} z_{i}+h\right)\right. \\
& \left.-\tanh \left(\beta\left(\frac{p}{2}\right)^{1 / 2} q^{(p-1) / 2} z_{i}+h\right)\right]^{2}, \\
\Gamma_{4}= & \mathbf{E}\left[\left\langle\sigma_{m+1}\right\rangle-\tanh \left(\beta\left(\frac{p}{2}\right)^{1 / 2} q^{(p-1) / 2} z_{m+1}+h\right)\right]^{2} .
\end{aligned}
$$


Lemma 3.1 for $a=1$ and symmetry yield $\Gamma_{1} \leq C / N$. The induction hypothesis implies the existence of independent standard Gaussian random variables $z_{1}, \ldots, z_{m}$ depending on $\left\{g_{i_{1}, \ldots, i_{p}} ;\left(i_{1}, \ldots, i_{p}\right) \in A_{N-1}^{p}\right\}$ such that $\Gamma_{2} \leq C(m, h) / N$. Using the inequality $|\tanh a-\tanh b| \leq|a-b|$ and Lemma 6.1 we obtain

$$
\Gamma_{3} \leq C\left[\left|\beta-\beta_{-}\right|+\left|q^{(p-1) / 2}-q_{-}^{(p-1) / 2}\right|\right] \leq C / N .
$$

Finally, Proposition 1.3 gives the existence of a standard Gaussian random variable $z=z_{m+1}$ such that $\Gamma_{4} \leq C(h) / N$ and $z_{m+1}$ is independent of $\left\{z_{1}, \ldots, z_{m}\right\}$ because these random variables depend only on $\left\{g_{i_{1}, \ldots, i_{p}}\right.$; $\left.\left(i_{1}, \ldots, i_{p}\right) \in A_{N-1}^{p}\right\}$.

\section{References}

X. Bardina, D. Márquez-Carreras, C. Rovira and S. Tindel (2004), The p-spin interaction model with external field, Potential Analysis 21, 311-362.

A. Bovier, I. Kurkova and M. Löwe (2002), Fluctuations of the free energy in the REM and the p-spin SK model, Ann. Probab. 30, 605-651.

A. Cadel, D. Márquez-Carreras and C. Rovira (2004), The Gibbs' measure in the p-spin interaction model with external field, Mathematics Preprint Series 356, http://www.imub. ub.es/publications/preprints/index2004.html.

E. Gardner (1985), Spin glasses with p-spin interactions, Nuclear Phys. B 257, 747-765.

D. Sherrington and S. Kirkpatrick (1975), Solvable model of a spin glass, Phys. Rev. Lett. 35, 1792-1796.

M. Talagrand (2000a), Rigorous low-temperature results for the mean field p-spins interaction model, Probab. Theory Related Fields 117, 303-360.

M. Talagrand (2000b), Spin Glasses: a Challenge for Mathematicians, Springer, Berlin, 2000 .

Facultat de Matemàtiques

Universitat de Barcelona

Gran Via 585, 08007 Barcelona, Spain

E-mail: davidmarquez@ub.edu

Received on 5.11.2006;

revised version on 17.1.2007 
